\title{
An Intensive Intervention to Reduce Readmissions for Frequently Hospitalized Patients: the CHAMP Randomized Controlled Trial
}

\author{
Bruce L. Henschen, MD, MPH' (D), Maria E. Theodorou, MD², Margaret Chapman, MD ${ }^{3}$, \\ McKay Barra, BA ${ }^{2}$, Abby Toms, MSW4, Kenzie A. Cameron, PhD, $\mathrm{MPH}^{7}$, \\ Shuhan Zhou, MPP2, Chen Yeh, MS ${ }^{5}$, Jungwha Lee, PhD, MPH', and Kevin J. O'Leary, \\ $M D, M S^{2}$
}

\begin{abstract}
'Division of General Internal Medicine and Geriatrics, Department of Medicine, Northwestern University Feinberg School of Medicine, Chicago, IL, USA; ${ }^{2}$ Division of Hospital Medicine, Department of Medicine, Northwestern University Feinberg School of Medicine, Chicago, IL, USA; ${ }^{3}$ Division of General Internal Medicine, Massachusetts General Hospital, Boston, MA, USA; ${ }^{4}$ Department of Social Work, Northwestern Memorial Hospital, Chicago, IL, USA; ${ }^{5}$ Division of Biostatistics, Department of Preventive Medicine, Northwestern University Feinberg School of Medicine, Chicago, IL, USA.
\end{abstract}

\begin{abstract}
BACKGROUND: A small number of patients are disproportionally readmitted to hospitals. The Complex High Admission Management Program (CHAMP) was established as a multidisciplinary program to improve continuity of care and reduce readmissions for frequently hospitalized patients.

OBJECTIVE: To compare hospital utilization metrics among patients enrolled in CHAMP and usual care.

DESIGN: Pragmatic randomized controlled trial.

PARTICIPANTS: Inclusion criteria were as follows: 3 or more, 30-day inpatient readmissions in the previous year; or 2 inpatient readmissions plus either a referral or 3 observation admissions in previous 6 months.

INTERVENTIONS: Patients randomized to CHAMP were managed by an interdisciplinary team including social work, physicians, and pharmacists. The CHAMP team used comprehensive care planning and inpatient, outpatient, and community visits to address both medical and social needs. Control patients were randomized to usual care and contacted 18 months after initial identification if still eligible.
\end{abstract}

MAIN MEASURES: Primary outcome was number of 30day inpatient readmissions 180 days following enrollment. Secondary outcomes were number of hospital admissions, total hospital days, emergency department visits, and outpatient clinic visits 180 days after enrollment.

KEY RESULTS: There were 75 patients enrolled in CHAMP, 76 in control. Groups were similar in demographic characteristics and baseline readmissions. At 180 days following enrollment, CHAMP patients had more inpatient 30-day readmissions [CHAMP incidence rate 1.3 (95\% CI 0.9-1.8) vs. control 0.8 (95\% CI 0.5-1.1), $p=0.04]$, though both groups had fewer readmissions

Prior Presentation This work was accepted as an oral presentation to the 2020 Society of General Internal Medicine National Meeting; however, due to the COVID-19 pandemic, we were unable to present our findings at the meeting and instead presented the work as a virtual poster. The work was also presented as an oral presentation at the Society of Hospital Medicine Converge 2021 National Meeting.

Received March 10, 2021

Accepted July 14, 2021

Published online September 1, 2021 compared to 180 days prior to enrollment. We found no differences in secondary outcomes.

CONCLUSIONS: Frequently hospitalized patients experienced reductions in utilization over time. Though most outcomes showed no difference, CHAMP was associated with higher readmissions compared to a control group, possibly due to consolidation of care at a single hospital. Future research should seek to identify subsets of patients with persistently high utilization for whom tailored interventions may be beneficial.

TRIAL REGISTRATION: ClinicalTrials.gov identifier: NCT03097640; https://clinicaltrials.gov/ct2/show/ NCT03097640

KEY WORDS: care transitions; readmissions; care models; continuity of care; randomized controlled trial.

J Gen Intern Med 37(8):1877-84

DOI: $10.1007 / \mathrm{s} 11606-021-07048-1$

(c) Society of General Internal Medicine 2021

small number of patients account for a disproportionate
number of hospital readmissions. ${ }^{1}$ While medically di-
verse, many patients who are frequently hospitalized have
behavioral or social needs that require holistic care models
emphasizing more than medical care alone. ${ }^{2}$ This population
challenges a system of care that fragments hospital-based care
and ambulatory care, creating systematic discontinuity for
patients who may require longitudinal relationship-based care
to meet their complex needs. ${ }^{3}$ In qualitative studies, patients
who are frequently hospitalized report frustration with care
fragmentation, causing them to perceive a lack of continuity
and a loss of trust with the medical system. ${ }^{4}$
Innovative care models have sought to reduce readmissions
through redesigning care delivery, improving care coordina-
tion, and connecting patients to existing resources. ${ }^{5-8}$ A sys-
tematic review of interventions for frequently hospitalized
patients found a heterogeneous group of care models. ${ }^{9}$ 
Importantly, the majority of studies were observational. Many patients experience a reduction in utilization after an initial period of frequent admissions, ${ }^{10}$ limiting the ability of observational studies to measure a specific program's effect due to the natural decline in readmissions over time. A randomized trial of a "healthcare hotspotting" intervention for patients in Camden, NJ, reported no change in hospitalization rates compared to a control group. ${ }^{11}$ Though this intervention was an intensive interdisciplinary effort that enrolled patients while still hospitalized, it focused primarily on connecting patients to existing outpatient resources. Other intensive outpatient-only interventions have failed to reduce healthcare utilization. ${ }^{12}$ Interventions that focus on improving care across clinical settings (i.e., both inside and outside of the hospital) may have a different effect.

We created the Complex High Admission Management Program (CHAMP) as a quality improvement initiative to improve inpatient and outpatient care and reduce inpatient readmissions of patients frequently admitted to our hospital. The CHAMP multidisciplinary team works to foster longitudinal relationships with patients who suffer from systematic discontinuity. A pilot pre-post analysis of CHAMP observed reductions in readmission, ${ }^{13}$ however, results may have been confounded by the aforementioned tendency for utilization to decline over time. ${ }^{10}$ In this study, we conducted a randomized trial of CHAMP compared with usual care to accurately assess the program's effect on hospital readmissions.

\section{METHODS}

\section{Setting and Intervention}

This study took place at Northwestern Memorial Hospital (NMH), a large, urban academic hospital that serves a diverse patient population in Chicago, IL. Started in 2015, CHAMP utilizes a relationship-based care model that seeks to improve care and reduce hospital readmissions for at-risk patients. At the time of this study, CHAMP consisted of two social workers (1.6 total full-time equivalent (FTE)), 2 physicians (0.6 total FTE), a program manager (0.1 FTE), data analyst (0.1 FTE), and pharmacy support from precepted residents provided by McGaw Medical Center, Chicago, IL. CHAMP is funded internally by NMH.

We queried the health system's Enterprise Data Warehouse (EDW) for patients who were admitted to the hospital and met the eligibility criteria listed below. This structured query language (SQL) query generated a report powered by Microsoft's SQL Server Reporting
Services (Microsoft Corp, Redmond, WA) and was deployed onto the health system's EDW portal. The CHAMP team accessed the EDW portal each weekday to identify potentially eligible patients.

Upon enrolling a new patient, the CHAMP team conducted an in-depth psychosocial assessment, helped the patient set specific health-related goals, and attempted to develop a therapeutic relationship (see Text Box 1 for further details of the CHAMP intervention). Over time, the team collaborated with patients to write a comprehensive care plan that was accessible to all clinicians throughout our health system. Components of the care plan included providing medical history and personal details that enhance each patient's individuality; care recommendations for the emergency department (ED), hospital, and community setting; and contact information for key team members. ${ }^{14}$ After enrollment, when a CHAMP patient arrived in the ED, a CHAMP team member received an automatic page, enabling them to contact the patient and ED team during business hours to help guide care. If hospitalized, one or more CHAMP team members visited each patient daily; CHAMP patients were directly cared for by hospital staff. CHAMP team members created and maintained the comprehensive care plan, coordinated follow-up care, and connected patients to existing community resources. These included, but were not limited to, housing support, behavioral health, access to food and medication, substance use treatment resources, referral to medical specialty services, home care, and transportation. After discharge, patients were scheduled to follow up with the CHAMP team in an outpatient clinic embedded in existing transitional care clinic space. ${ }^{15} \mathrm{We}$ held clinics twice weekly, allowing for flexible scheduling. Some CHAMP patients used this clinic as their primary care physician (PCP) medical home, while others continued to see their existing PCP. In these cases, patients collaborated on a care plan and were followed primarily by the CHAMP team's social workers. CHAMP social workers frequently made outreach to patients beyond the inpatient and primary care setting, accompanying patients to subspecialty medical visits to provide support and help patients navigate follow-up. CHAMP social workers further conducted community visits for patients in settings such as state offices to help complete disability paperwork. Finally, CHAMP team members made ad hoc visits to nursing homes and other local health care facilities when necessary. These efforts attempted to coordinate care across health care settings, engage patients beyond traditional clinical environments, and build trust. 
Text Box 1 Complex High Admission Management Program (CHAMP) intervention details

\begin{tabular}{|c|c|}
\hline Inpatient & Emergency Department (ED) \\
\hline $\begin{array}{l}\text { - Identify and meet new patients } \\
\text { - } \quad \text { Develop individualized care plans } \\
\text { - Conduct daily team huddle and } \\
\text { routine inpatient "rounds" } \\
\text { - Hospital teams care directly for } \\
\text { hospitalized patients } \\
\text { - Conduct care planning and } \\
\text { coordination }\end{array}$ & $\begin{array}{l}\text { - Receive alert when patients arrive to } \\
\text { ED } \\
\text { - See patients in ED when necessary } \\
\text { - Attempt to divert patients to clinic } \\
\text { when appropriate/feasible } \\
\text { - Support patient and ED care team }\end{array}$ \\
\hline Clinic & Community \\
\hline $\begin{array}{l}\text { - Hold two clinics per week } \\
\text { - Physician, social work, pharmacy visit } \\
\text { patient together } \\
\text { - Utilize flexible scheduling model with } \\
\text { variable appointment times } \\
\text { - Select patients hold "drop-in" } \\
\text { privileges }\end{array}$ & $\begin{array}{l}\text { - Conduct pharmacy visits for } \\
\text { medication teaching } \\
\text { - Visit government benefits offices } \\
\text { - Attend outpatient specialty } \\
\text { appointments with patients } \\
\text { - Visit patients at outpatient facilities, } \\
\text { when possible }\end{array}$ \\
\hline
\end{tabular}

\section{Study Design}

CHAMP-eligible patients started as those with 3 or more 30-day all-cause all-payer unplanned inpatient readmissions, as defined by Medicare, to $\mathrm{NMH}$ in the past year. ${ }^{16}$ Prior to this study, the CHAMP team enrolled 51 patients, who did not contribute data to this RCT but were cared for by the CHAMP team concurrently, which affected team capacity. For this study, qualifying patients were randomized in a 1:1 fashion to the CHAMP intervention or a delayed-enrollment control group. We experienced slowing recruitment as the trial progressed. In response, we opted to expand inclusion criteria to 2 or more readmissions plus either 3 observation admissions in the prior 6 months or a referral from the primary care team. We expanded inclusion criteria to achieve an adequate sample size; an a priori power calculation based on pre-post CHAMP pilot data, in which we observed a $40 \%$ reduction in readmissions, ${ }^{13}$ determined 176 total patients were necessary to detect a $40 \%$ difference in readmissions between groups using thresholds of $\alpha<0.05$ and $\beta>0.8$. Patients were excluded if they already were engaged with a multidisciplinary team (e.g., transplant patients, those with malignancy, or those enrolled in a simultaneous readmissions program for heart failure patients) or if they were admitted to a non-medicine service line (e.g., surgery, psychiatry) or to the intensive care unit. We excluded pregnant women, those currently incarcerated, non-English speakers, and minors.

Eligible patients were randomized using permuted blocks with random block sizes between 4 and 8 generated in STATA (StataCorp, College Station, TX), stratified by sex and sickle cell disease. As they were part of the CHAMP team, investigators were blinded to the allocation sequence but not an eventual group assignment. We stratified by sickle cell disease as approximately one-third of our original CHAMP patients carried this diagnosis and their care may be unique. ${ }^{17}$ Patients randomized to CHAMP were approached by a member of the CHAMP team during a hospital admission and invited to enroll in the program. Patients randomized to the control group received usual care during the study; they were invited 
to participate in the CHAMP quality improvement effort as a non-study patient if they still met inclusion criteria 18 months after their randomization date.

\section{Outcomes and Data Analysis}

Our primary outcome was the number of 30-day unplanned inpatient readmissions to $\mathrm{NMH}$ at 180 days after enrollment. Secondary outcomes included 30-day inpatient readmissions measured at both 30 and 90 days; total hospital admissions (inpatient and observation status), emergency department (ED) discharges, total outpatient clinic visits, and total number of days hospitalized at NMH 180 days post-enrollment. We obtained these data, and information on comorbidities and mortality, using the health system's EDW. We used the Area Deprivation Index (ADI) - a validated estimate of socioeconomic disparity in geographic areas, wherein higher scores correspond to a higher burden of social determinants of health - in each participant's neighborhood. ${ }^{18,19}$ We collected data on the intervention's fidelity-including measures of continuity (number of interactions with the CHAMP team, number of clinic and hospital visits) and number of care plans developed - using the EDW and limited chart reviews.

Descriptive statistics were calculated for all variables of interest. Categorical variables were summarized with the use of counts and percentages; continuous variables were measured using median with range or mean with standard deviation (SD). To examine the relationship between intervention and outcome of interest, we performed Poisson regression models for count data except for total number of hospital days and mortality. For Poisson regression models, we reported incidence rates (IRs) and incidence rate ratios (IRR) comparing CHAMP and control and corresponding 95\% CIs. For the total number of hospital days, we used median quantile regression and reported median difference (95\% CI) between CHAMP and control. For mortality, we used a logistic regression model and reported odds ratios (OR) and corresponding $95 \% \mathrm{CI}$. We analyzed all data according to the intent-to-treat principle; participants were analyzed by group assignment regardless of their degree of participation in CHAMP. All analyses were conducted in SAS version 9.4 (the SAS Institute; Cary, NC) and R 4.0.1. This study was approved by the Northwestern University Institutional Review Board (STU00203847) and registered at Clinicaltrials.gov prior to enrolling patients.

\section{RESULTS}

\section{Patient Characteristics}

We randomized 151 patients from October 1, 2016, to June 13, 2019; enrollment was stopped on this date due to a slowing of eligible patients and a turnover in the program's social work staff. Figure 1 shows participant flow through the study. Baseline characteristics of enrolled patients were balanced between intervention and control groups. Both groups contain a high prevalence of chronic diseases, including chronic pain (80\% in CHAMP, $87 \%$ in control). Groups had similar burdens of social determinants of health as estimated by the ADI (Table 1).

Out of the 75 patients enrolled in CHAMP, 7 patients either declined to meet the CHAMP team or were approached by the team but subsequently lost to follow-up. An

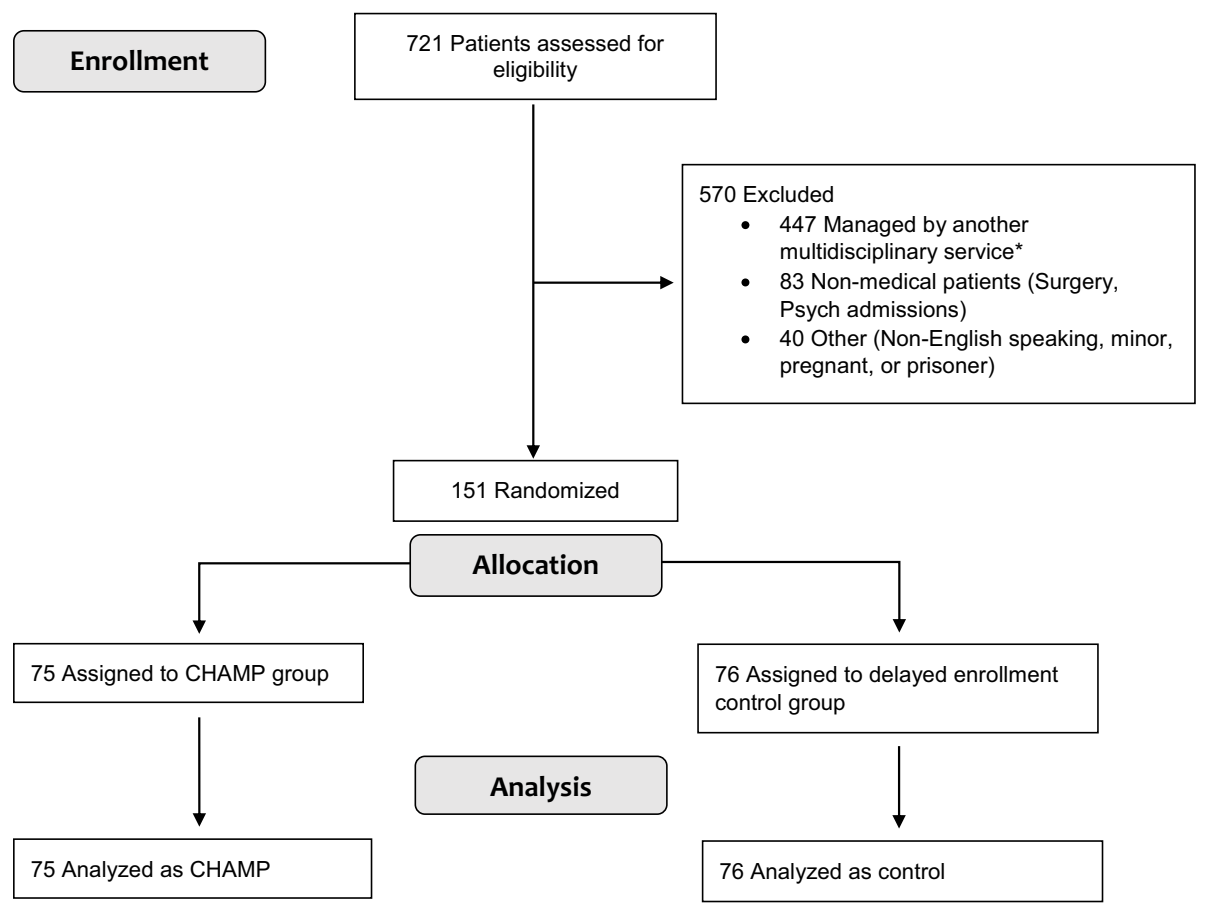

Figure 1 Flow of participants in the study. *Multidisciplinary services include the following: oncology, Heart Failure Bridget and Transition (BAT) team, transplant, cystic fibrosis, infectious disease/HIV care, hospice. 
Table 1 Demographics and Baseline Characteristics of Study Participants

\begin{tabular}{|c|c|c|}
\hline Characteristic & CHAMP $(N=75)$ & Control $(N=76)$ \\
\hline Age at enrollment, mean (SD) & $52.8(17.3)$ & $54.4(16.5)$ \\
\hline Female, $n(\%)$ & $40(53 \%)$ & $40(53 \%)$ \\
\hline \multicolumn{3}{|l|}{ Comorbidity, $n(\%)$} \\
\hline Chronic pain ( $>1$ opioid prescription during study) & $60(80 \%)$ & $65(86 \%)$ \\
\hline Substance use disorder & $42(56 \%)$ & $32(42 \%)$ \\
\hline COPD & $39(52 \%)$ & $39(51 \%)$ \\
\hline $\mathrm{CHF}$ & $37(49 \%)$ & $42(55 \%)$ \\
\hline Chronic kidney disease (any stage) & $36(48 \%)$ & $44(58 \%)$ \\
\hline Prescribed SSRI/SNRI during study period & $27(36 \%)$ & $19(25 \%)$ \\
\hline Sickle cell disease & $7(9 \%)$ & $6(8 \%)$ \\
\hline \multicolumn{3}{|l|}{ Insurance status at enrollment $t^{\dagger}$} \\
\hline Medicaid/Medicaid replacement only & $17(23 \%)$ & $20(26 \%)$ \\
\hline Medicare/Medicare advantage only & $26(34 \%)$ & $32(42 \%)$ \\
\hline Dual-eligible (Medicare/Medicaid) & $5(7 \%)$ & $7(9 \%)$ \\
\hline Private insurance & $15(20 \%)$ & $8(11 \%)$ \\
\hline No insurance identified & $27(36 \%)$ & $27(36 \%)$ \\
\hline \multicolumn{3}{|l|}{ Other social determinants } \\
\hline Self-identified as Black/Indigenous/person of Color (BIPOC) & $46(61 \%)$ & $44(58 \%)$ \\
\hline Self-identified as Hispanic & $6(8 \%)$ & $6(8 \%)$ \\
\hline Area Deprivation Index, national percentile (range) ${ }^{\ddagger}$ & $45(2-93)$ & $53(2-99)$ \\
\hline
\end{tabular}

Abbreviations: CHF, congestive heart failure; COPD, chronic obstructive pulmonary disease; SSRI, selective serotonin reuptake inhibitor; SNRI, serotonin-norepinephrine reuptake inhibitor

*Determined by presence of ICD-10 code for substance use disorders or the prescription of either buprenorphine or methadone during the study period ${ }^{+}$Columns do not add to $100 \%$ as patients can report multiple types of insurance at one time

${ }^{*}$ An estimate of socioeconomic disparity based on zip code listed at enrollment; higher scores correspond to a higher degree of deprivation

additional 12 patients agreed to meet the team and had a "placeholder" placed in their chart but did not receive a care plan. The most common reasons for not receiving a care plan were either that the patient died within 30 days or had a primary care physician who opted not to have the CHAMP team involved. The remaining 56 patients $(75 \%)$ received an individualized care plan. Table 2 displays data summarizing the fidelity of implementing the components of the CHAMP intervention. CHAMP patients with a care plan had an average of 17 contacts with a CHAMP team member, including inpatient visits, outpatient clinic visits, community visits, and phone calls. Among CHAMP patients with a care plan, a CHAMP team member visited them during 93\% of inpatient hospitalizations (141/151). Among this same group, 48\% (27/56) attended CHAMP clinic at least

Table 2 Fidelity of Implementing the CHAMP Intervention Among Patients Enrolled in the Intervention Arm

\begin{tabular}{ll}
\hline \hline Metric & CHAMP \\
\hline Number of patients with care plan developed, $n(\%)$ & \\
Within 30 days & $14(19 \%)$ \\
Within 60 days & $36(48 \%)$ \\
Within 90 days & $53(71 \%)$ \\
Within 180 days & $56(75 \%)$ \\
For those patients with a care plan $(n=56)$ & $4.8(4.9)$ \\
Number of care plan updates within 180 days, mean & \\
(SD) & 17.0 \\
Number of CHAMP team contacts within 180 days, & $(10.2)$ \\
mean (SD) & \\
Number of completed clinic appointments within 180 & $1.5(2.4)$ \\
days, mean (SD) & \\
\hline
\end{tabular}

${ }^{*}$ CHAMP team contacts included visits with a social worker, and/or physician, in the inpatient, outpatient, or community setting; as well as phone calls for which there was documentation in the medical record once; the overall clinic show rate (appointments completed/appointments made) was $70 \%$ (86/122).

\section{Primary Outcome}

CHAMP and control patients experienced similar rates of 30day inpatient readmission during the 180 days prior to enrollment in the study (Table 3). At the end of the study period, readmission rates were lower than baseline in both CHAMP

Table 3 Utilization Outcomes Among Patients Enrolled in the Study

\begin{tabular}{|c|c|c|c|}
\hline Utilization outcome $^{*}$ & $\begin{array}{l}\text { CHAMP } \\
(N=75)\end{array}$ & $\begin{array}{l}\text { Control } \\
(N=76)\end{array}$ & $\begin{array}{l}\text { IRR }[95 \% \\
\text { CI], } p_{-}^{-} \\
\text {value }^{\dagger}\end{array}$ \\
\hline $\begin{array}{l}\text { Inpatient, 30-day read- } \\
\text { missions for } 180 \text { days } \\
\text { prior to study (baseline) }\end{array}$ & $\begin{array}{l}2.68[2.32- \\
3.10]\end{array}$ & $\begin{array}{l}2.72 \\
{[2.36-} \\
3.14]\end{array}$ & $\begin{array}{l}0.98[0.80 \\
1.21], 0.88\end{array}$ \\
\hline $\begin{array}{l}\text { Inpatient, } 30 \text {-day read- } \\
\text { missions at } 180 \text { days fol- } \\
\text { lowing study enrollment }\end{array}$ & $\begin{array}{l}1.29[0.94 \\
1.77]\end{array}$ & $\begin{array}{l}0.75 \\
{[0.50-} \\
1.13]\end{array}$ & $\begin{array}{l}1.72[1.03, \\
2.90], 0.04\end{array}$ \\
\hline $\begin{array}{l}\text { All hospitalizations at } \\
180 \text { days }^{\S}\end{array}$ & $\begin{array}{l}2.47[1.95- \\
3.11]\end{array}$ & $\begin{array}{l}1.99 \\
{[1.53-} \\
2.57]\end{array}$ & $\begin{array}{l}1.24[0.88, \\
1.76], 0.22\end{array}$ \\
\hline $\begin{array}{l}\text { Emergency Department } \\
\text { discharges at } 180 \text { days }\end{array}$ & $\begin{array}{l}0.55[0.34 \\
0.87]\end{array}$ & $\begin{array}{l}0.68 \\
{[0.45-} \\
1.04]\end{array}$ & $\begin{array}{l}0.80[0.43, \\
1.49], 0.48\end{array}$ \\
\hline $\begin{array}{l}\text { Outpatient clinic visits at } \\
180 \text { days }\end{array}$ & $\begin{array}{l}5.45[4.18- \\
7.11]\end{array}$ & $\begin{array}{l}4.18 \\
{[3.10-} \\
5.65]\end{array}$ & $\begin{array}{l}1.30[0.87, \\
1.95], 0.20\end{array}$ \\
\hline $\begin{array}{l}\text { Total hospital days at } 180 \\
\text { days following } \\
\text { enrollment, median [95\% } \\
\text { CI] }\end{array}$ & $\begin{array}{l}9.00[5.21- \\
12.79]\end{array}$ & $\begin{array}{l}10.00 \\
{[3.20-} \\
16.80]\end{array}$ & 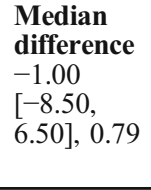 \\
\hline \multicolumn{4}{|c|}{$\begin{array}{l}\text { Incidence rate for outcomes [95\% CI] from Poisson regression except } \\
\text { for total hospital days where median [95\% CI] from median quantile } \\
\text { regression are reported by groups } \\
\text { Incidence rate ratio (IRR) comparing outcomes between CHAMP and } \\
\text { control } \\
\text { Denotes primary outcome }\end{array}$} \\
\hline
\end{tabular}


and control groups. Despite this decrease, at 180 days following enrollment we found a higher rate of 30-day readmissions in the CHAMP intervention group compared to control (CHAMP IR 1.29 [95\% CI 0.94-1.77] vs. control 0.75 [0.50-1.13], IRR 1.72 [1.03-2.90], $p=0.04)$. In the study period, there were 97 readmissions in CHAMP (range 0-13 per patient) and 55 readmissions in control (range 0-6). Recruitment of patients with sickle cell disease was smaller than expected ( $n=7$ in CHAMP, 6 in control); thus, we opted not to analyze this subgroup. When measuring patients without sickle cell disease, the rates of inpatient readmissions at 180 days were smaller (CHAMP IR 0.87 [95\% CI 0.61-1.24] vs. control 0.67 [0.45-1.00]; IRR 1.29 [0.76-2.20], $p=0.34$ ). Within this subgroup, there were 59 readmissions in CHAMP (range $0-7$ ) compared to 47 in control (range $0-6$ ).

\section{Secondary Outcomes}

We did not observe differences in rates of 30-day readmissions measured at 30 days (CHAMP IR 0.25 [95\% CI 0.17-0.38] vs. control 0.16 [0.10-0.26], IRR 1.60 [0.84-3.05], $p=0.15$ ) or 90 days post-enrollment (CHAMP IR 0.77 [95\% CI 0.551.09 ] vs. control 0.50 [0.33-0.77], IRR 1.55 [0.89-2.68], $p=0.12$ ). There were no differences between groups in the number of hospital admissions, total hospital days, emergency department visits, and outpatient clinic visits 180 days after enrollment (Table 3). Overall mortality between the two groups was not significantly different (odds ratio CHAMP vs. control $0.81,95 \%$ CI $0.242 .78, p=0.73$ ).

\section{DISCUSSION}

In this pragmatic randomized controlled trial, we found that patients assigned to a high-intensity, multidisciplinary care model experienced more readmissions at 180 days compared to the group receiving usual care, though both groups experienced a decline in readmissions after enrollment. CHAMP may have been ineffective at reducing readmissions for several reasons. First, the program offers patients a stable medical home in a city with many options for health care. Patients may have elected to consolidate care at our institution due to the unique opportunity to see the same multidisciplinary team members at every inpatient admission and in the outpatient clinic. We may have succeeded in increasing continuity among these patients, which could have resulted in increased health care utilization at our institution. Other interventions that facilitate access to high-quality medical care have also demonstrated increases in utilization, demonstrating a potential unmet need for medical or social services among vulnerable groups. ${ }^{20,}{ }^{21}$ Second, anecdotal reports of individual successes often come after months, or years, of work on patient activation, trust-building, and learning from prior failed attempts. ${ }^{14}$ A longer time frame may be needed to detect benefits for certain patients. Third, as demonstrated in observational studies, identifying patients at "peak utilization" may overlook the natural tendency for utilization to decline. ${ }^{10}$ Patients in our study may have been poised to experience a reduction in utilization, regardless of their randomization to CHAMP or usual care. The majority of CHAMP patients received a care plan and had frequent contacts with the intervention clinical team, suggesting our findings are not related to poor fidelity of implementation.

Our findings raise important questions about the optimal approach to improve care for patients who are frequently hospitalized. While utilization is an important metric for program success, it is not the only clinically relevant metric. Though utilization may not change, CHAMP - and other interventions that foster patient continuity and connect patients to medical and social services (e.g., housing support, access to food or medication) - may improve patient satisfaction, trust in the medical system and, perhaps, quality of life. ${ }^{22-24}$ Further research should examine patient-reported outcomes, addressing social determinants of health, and physician and staff perceptions. Studies should seek to capture health-related social needs in a systematic and more comprehensive way and develop systems-level approaches to address these needs.

Prior studies of similar interventions, including those focused on home-based, interdisciplinary care, and comprehensive care planning also found no difference in rates of hospital

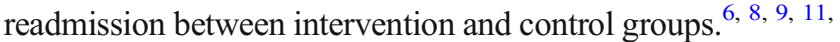
${ }^{25,}{ }^{26}$ Comparing our study with these, there is clear heterogeneity in who qualifies as a "high utilizer" patient. Within this diverse group, there are likely subsets of patients whose utilization will decline without any intervention; those whose utilization will not decline with any intervention; and those for whom a program like CHAMP might be beneficial. In qualitative studies, we have found that patients identify psychological, social, and economic stressors as triggers for hospitalization and adverse health outcomes. ${ }^{27}$ While our intervention was designed to enhance continuity of care, build trust, and connect patients with existing community resources, patients' psychological, social, and economic vulnerabilities remain challenging to address. Based on our findings, and those from prior studies, interventions that focus on patients with particular needs, such as housing, financial stability, or need for psychosocial and mental health services, may be more effective. ${ }^{28-32}$ Alternatively, disease-specific interventions - such as those for sickle cell disease - or those focused on moderate-risk patients with more limited needs may be fruitful. ${ }^{33,}{ }^{34}$ Further research should seek to identify unique needs within this heterogeneous group and work to target interventions to subgroups of patients most likely to benefit.

Our study's strengths included a randomized design pragmatically embedded in an existing quality improvement program with a lengthy follow-up period. However, our study had several limitations. First, we were underpowered to detect our primary outcome due to slower-than-expected enrollment and changes in the team structure. A larger study would have enabled examination of subgroups and additional secondary outcomes, such as cost. Second, the EDW used to gather data 
may be an inadequate tool to accurately capture data such as social determinants of health, completion of referrals to community or social support services, or capturing utilization, or deaths occurring outside of Northwestern Medicine. Our use of surrogate measures of social determinants may have underestimated the burden of social needs in this population. Third, some patients in the CHAMP arm did not end up having a care plan published. In many of these instances, the time required to build the relationship and patient-clinician trust necessary to publish a care plan were longer than the brief 180-day study period. In this pragmatic trial with intention-to-treat analysis, we analyzed these patients as if they received the full intervention. Finally, the intensive nature of the CHAMP intervention may be difficult to scale across a broader health system or population.

Overall, the CHAMP intervention did not result in fewer readmissions when compared to a concurrent control group receiving usual care. Future research should determine the effect of similar interventions on quality of life and patientreported outcomes and seek to more systematically and comprehensively capture health-related social needs. Reliable methods to predict future utilization based on comorbidities and social needs would help identify which populations may be at early risk of high hospital use. Such efforts could lead to more precise direction of interventions toward subgroups of patients most likely to benefit.

Acknowledgements: The authors would like to acknowledge Luke Hansen, MD, and Suzanne Condon-Paskiewicz, LCSW, CCM, for their instrumental role in growing CHAMP. The authors also acknowledge Emily Wilson, MBA, MHA, Claire Knoten, PhD, Erin Lambers, PhD, MPH, and Shandu Foster, MA, for their administrative support during the trial. Finally, the authors thank Gary Noskin, MD, and Julie Creamer, MS, RN, for their ongoing institutional and financial support of CHAMP.

Corresponding Author: Bruce L. Henschen, MD, MPH; Division of General Internal Medicine and Geriatrics, Department of Medicine, Northwestern University Feinberg School of Medicine, Chicago, IL, USA (e-mail: b-henschen@northwestern.edu).

Funding Internal funding for this study was provided by the Division of Hospital Medicine, Northwestern University Feinberg School of Medicine, Chicago, IL.

\section{Declarations:}

Conflict of Interest: The authors declare that they do not have a conflict of interest.

\section{REFERENCES}

1. Szekendi MK, Williams MV, Carrier D, Hensley L, Thomas S, Cerese J. The characteristics of patients frequently admitted to academic medical centers in the United States. J Hosp Med. 2015;10(9):563-568.

2. Rinehart DJ, Oronce C, Durfee MJ, et al. Identifying Subgroups of Adult Superutilizers in an Urban Safety-Net System Using Latent Class Analysis: Implications for Clinical Practice. Med Care. 2018;56(1):e1-e9.

3. Hochman M, Asch SM. Disruptive Models in Primary Care: Caring for High-Needs, High-Cost Populations, J Gen Intern Med. 2017;32(4):392397.
4. Mautner DB, Pang H, Brenner JC, et al. Generating hypotheses about care needs of high utilizers: lessons from patient interviews. Popul Health Manag. 2013;16 Suppl 1:S26-33.

5. Mercer T, Bae J, Kipnes $\mathbf{J}$, Velazquez M, Thomas S, Setji N. The highest utilizers of care: individualized care plans to coordinate care, improve healthcare service utilization, and reduce costs at an academic tertiary care center. J Hosp Med. 2015;10(7):419-424.

6. Weerahandi $\mathbf{H}$, Basso Lipani $\mathbf{M}$, Kalman $\mathbf{J}$, et al. Effects of a Psychosocial Transitional Care Model on Hospitalizations and Cost of Care for High Utilizers. Soc Work Health Care. 2015;54(6):485-498.

7. Lynch CS, Wajnberg A, Jervis $\mathbf{R}$, et al. Implementation Science Workshop: a Novel Multidisciplinary Primary Care Program to Improve Care and Outcomes for Super-Utilizers. J Gen Intern Med. 2016;31(7):797-802.

8. Zulman DM, Pal Chee C, Ezeji-Okoye SC, et al. Effect of an Intensive Outpatient Program to Augment Primary Care for High-Need Veterans Affairs Patients: A Randomized Clinical Trial. JAMA Intern Med. 2017;177(2):166-175.

9. Goodwin A, Henschen BL, O'Dwyer LC, Nichols N, O'Leary KJ. Interventions for Frequently Hospitalized Patients and Their Effect on Outcomes: A Systematic Review. J Hosp Med. 2018;13(12):853-859.

10. Johnson TL, Rinehart DJ, Durfee $\mathbf{J}$, et al. For many patients who use large amounts of health care services, the need is intense yet temporary. Health Aff. 2015;34(8):1312-1319.

11. Finkelstein A, Zhou A, Taubman S, Doyle J. Health Care Hotspotting A Randomized, Controlled Trial, N Engl J Med. 2020;382(2):152-162.

12. Edwards ST, Peterson K, Chan B, Anderson J, Helfand M. Effectiveness of Intensive Primary Care Interventions: A Systematic Review. J Gen Intern Med. 2017 Dec;32(12):1377-1386. doi: https://doi.org/10.1007/ s11606-017-4174-Z.

13. Henschen BL, Chapman M, Toms A, Barra M, Hansen LO. The Complex High Admission Management Program (CHAMP): Development and Preliminary Impact on Hospital Utilization. . J Gen Intern Med. 2017;32(2): S799-S800.

14. Theodorou ME, Henschen BL, Chapman M. The Comprehensive Care Plan: A Patient-Centered, Multidisciplinary Communication Tool for Frequently Hospitalized Patients. Jt Comm J Qual Patient Saf. 2020;46(4):217-226

15. Liss DT, Ackermann RT, Cooper A, et al. Effects of a Transitional Care Practice for a Vulnerable Population: a Pragmatic, Randomized Comparative Effectiveness Trial. J Gen Intern Med. 2019;34(9):1758-1765.

16. Center for Medicare and Medicaid Services. 2015 Measure information about the 30-day all cause hospital readmission measure, calculated for the value-based payment modifier program. Marcy 2017. https://www. cms.gov/Medicare/Medicare-Fee-for-Service-Payment/PhysicianFeedbackProgram/Downloads/2015-ACR-MIF.pdf. Accessed June 1, 2021.

17. Koch KL, Karafin MS, Simpson P, Field JJ. Intensive management of high-utilizing adults with sickle cell disease lowers admissions. Am J Hematol. 2015;90(3):215-219.

18. Kind AJH, Buckingham W. Making Neighborhood Disadvantage Metrics Accessible: The Neighborhood Atlas. N Engl J Med, 2018;378:2456-2458.

19. University of Wisconsin School of Medicine Public Health. 2015 Area Deprivation Index, v2.0. May 23, 2019. https://www.neighborhoodatlas. medicine.wisc.edu/. Accessed June 26, 2021.

20. Baicker K, Taubmab SL, Allen HL, et al. The Oregon Experiment Effects of Medicaid on Clinical Outcomes. N Engl J Med. 2013; 368(18): 1713-1722

21. Farrell CM, Gottlieb A. The Effect of Health Insurance on Health Care Utilization in the Justice-Involved Population: United States, 2014-2016. Am J Public Health. 2020; 110(Suppl 1): S78-S84.

22. Levine DM, Landon BE, Linder JA. Quality and Experience of Outpatient Care in the United States for Adults With or Without Primary Care. JAMA Intern Med. 2019;179(3):363-372.

23. Zulman DM, Chang ET, Wong A, et al. Effects of Intensive Primary Care on High-Need Patient Experiences: Survey Findings from a Veterans Affairs Randomized Quality Improvement Trial. J Gen Intern Med. 2019;34(Suppl 1):75-81.

24. Wu F, Slightam CA, Wong A, Asch S, Zulman DM. Intensive Outpatient Program Effects on High-need Patients' Access, Continuity, Coordination, and Engagement. Med Care. 2018;56(1):19-24.

25. Plant NA, Kelly PJ, Leeder SR, et al. Coordinated care versus standard care in hospital admissions of people with chronic illness: a randomised controlled trial. Med J Australia 2015;203(1):33-39.

26. Shepstone, L., Lipp, A., Christou, M., Evans, D., Hand, C. (2005). Does home based medication review keep older people out of hospital? The 
HOMER randomised controlled trial. BMJ (Clinical research ed.), 330(7486), 293.

27. O'Leary KJ, Chapman MM, Foster S, O'Hara L, Henschen BL, Cameron KA. Frequently Hospitalized Patients' Perceptions of Factors Contributing to High Hospital Use. J Hosp Med. 2019;14:E1-E6.

28. Kangovi S, Mitra N, Norton L, et al. Effect of Community Health Worker Support on Clinical Outcomes of Low-Income Patients Across Primary Care Facilities: A Randomized Clinical Trial. JAMA Intern Med. 2018;178(12):1635-1643.

29. Victor RG, Lynch K, Li N, et al. A Cluster-Randomized Trial of BloodPressure Reduction in Black Barbershops. $N$ Engl $J$ Med. 2018;378(14): 1291-1301.

30. Levine DM, Ouchi K, Blanchfield B, et al. Hospital-Level Care at Home for Acutely Ill Adults: A Randomized Controlled Trial. Ann Intern Med. 2020;172(2):77-85.

31. Sadowski LS, Kee RA, VanderWeele TJ, Buchanan D. Effect of a housing and case management program on emergency department visits and hospitalizations among chronically ill homeless adults: a randomized trial. JAMA. 2009:301(17):1771-1778.

32. Tanmoy Das L, Abramson EL, Kaushal R. High-Need, High-Cost Patients Offer Solutions for Improving Their Care and Reducing Costs. NEJM Catayst. 2019. https://catalyst-nejm-org.ezproxy.galter.northwestern.edu/high-need-high-cost-patients-solutions/. Accessed March 1, 2021.

33. Balsamo L, Shabanova V, Carbonella J, et al. Improving Care for Sickle Cell Pain Crisis Using a Multidisciplinary Approach. Pediatrics. 2019;143(5):e20182218.

34. Lanzkron S, Carroll CP, Hill P, David M, Paul N, Haywood C, Jr. Impact of a dedicated infusion clinic for acute management of adults with sickle cell pain crisis. Am J Hematol. 2015;90(5):376-380.

Publisher's Note: Springer Nature remains neutral with regard to jurisdictional claims in published maps and institutional affiliations. 